Check for updates

Cite this: Phys. Chem. Chem. Phys., 2019, 21, 3734

Received 4th September 2018, Accepted 5th November 2018

DOI: 10.1039/c8cp05604h

rsc.li/pccp

\section{Interfacial premelting of ice in nano composite materials $\dagger$}

\author{
Hailong Li, (D) a Markus Bier, (DD bcg Julian Mars, (D) ad Henning Weiss, ${ }^{\text {a }}$ \\ Ann-Christin Dippel, iD e Olof Gutowski, ${ }^{e}$ Veijo Honkimäki ${ }^{f}$ and Markus Mezger (D) *ad
}

The interfacial premelting in ice/clay nano composites was studied by high energy X-ray diffraction. Below the melting point of bulk water, the formation of liquid water was observed for the ice/ vermiculite and ice/kaolin systems. The liquid fraction is gradually increasing with temperature. For both minerals, similar effective premelting layer thicknesses of 2-3 nm are reached $3 \mathrm{~K}$ below the bulk melting point. For the quantitative description of the molten water fraction in wet clay minerals we developed a continuum model for short range interactions and arbitrary pore size distributions. This model quantitatively describes the experimental data over the entire temperature range. Model parameters were obtained by fitting using a maximum entropy (MaxEnt) approach. Pronounced differences in the deviation from Antonow's rule relating interfacial free energy between ice, water, and clay are observed for the charged vermiculite and uncharged kaolin minerals. The resultant parameters are discussed in terms of their ice nucleation efficiency. Using well defined and characterized ice/clay nano composite samples, this work bridges the gap between studies on single crystalline ice/solid model interfaces and naturally occurring soils and permafrost.

\section{Introduction}

Interface induced premelting of ice has important implications for environmental and technical processes. The formation of this liquid water layer at ice/solid interfaces contributes to the low friction observed on ice surfaces. ${ }^{1}$ In addition, most of the fresh water on earth is contained in ground ice and permafrost soil. The material properties of these composites strongly depend on the ice/solid interfacial properties. ${ }^{2}$ Already in 1859, Faraday proposed the existence of a liquid-like layer at ice surfaces. ${ }^{3}$ However, despite its importance and the amount of research devoted to the understanding of the surface and

\footnotetext{
${ }^{a}$ Max Planck Institute for Polymer Research, Ackermannweg 10, 55128 Mainz, Germany. E-mail: mezger@mpip-mainz.mpg.de

${ }^{b}$ Max Planck Institute for Intelligent Systems, Heisenbergstr. 3, 70569 Stuttgart, Germany

${ }^{c}$ Institute for Theoretical Physics IV, University of Stuttgart, Pfaffenwaldring 57, 70569 Stuttgart, Germany

${ }^{d}$ Institute of Physics, Johannes Gutenberg University Mainz, Staudingerweg 10, 55128 Mainz, Germany

${ }^{e}$ Deutsches Elektronen-Synchrotron DESY, Notkestr. 85, 22607 Hamburg, Germany

${ }^{f}$ ESRF-The European Synchrotron, Avenue des Martyrs 71, 38043 Grenoble Cedex 9, France

${ }^{g}$ Faculty of Applied Natural Sciences and Humanities, University of Applied Sciences Würzburg-Schweinfurt, Ignaz-Schön-Straße 11, 97421 Schweinfurt, Germany

$\dagger$ Electronic supplementary information (ESI) available: Sample characterization, experimental details and data processing. See DOI: $10.1039 / \mathrm{c} 8 \mathrm{cp} 05604 \mathrm{~h}$
}

interfacial premelting of ice, the formation mechanism of this liquid layer is still under debate. ${ }^{4-6}$

As of today, different physical effects contributing to the interfacial premelting phenomenon have been identified. ${ }^{5,7}$ In ice composites, the most relevant ones are the intrinsic interfacial melting, impurities, confinement, and geometry effects (Fig. 1). For non-planar interfaces such as in ice nano-crystals, ${ }^{8}$ nano-pores, ${ }^{9}$ and spherical nano-powders ${ }^{10}$ the geometry of the ice/water phase boundary plays an important role. Here, the Gibbs-Thomson effect leads to a melting point depression proportional to the mean curvature of the phase boundary. ${ }^{7}$ Confinement leads to a hysteresis in the freezing and melting process. ${ }^{11,12}$ Most impurities such as ions have a lower solubility in ice compared to liquid water. These impurities will be expelled

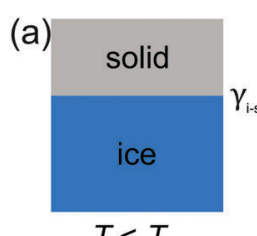

$T<T_{0}$

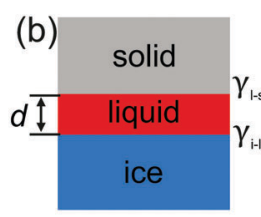

$T_{0}<T \leq T_{\mathrm{m}}$

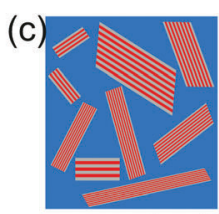

permafrost
Fig. 1 Sketch of premelting at ice/solid interfaces. (a) Ideal ice/solid interface with interfacial tension $\gamma_{i-s}$. (b) Above the onset temperature $T_{0}$, a thin premelting layer of thickness $d$ is formed before bulk melting sets in at $T_{m}$. (c) In natural ice/solid composite materials such as permafrost, the premelting mechanism is also affected by impurities, confinement and the geometry of the system. 
from the ice phase. Their concentration in the remaining liquid phase will therefore gradually lower its melting point. ${ }^{13}$ Finally, the intrinsic interfacial premelting and the grain boundary melting of a material are governed by the thickness dependent balance between the ice/liquid and the liquid/solid interfacial tension. $^{14}$

Since the first observations by Faraday, several experimental techniques have been employed to disentangle these contributions to the premelting mechanism for different ice interfaces. Well defined, planar, single crystalline ice surfaces and ice/solid interfaces were investigated by glancing angle X-ray scattering, ${ }^{15}$ X-ray reflectivity, ${ }^{16-18}$ X-ray photoemission spectroscopy, ${ }^{19}$ sum frequency generation spectroscopy, ${ }^{20}$ and molecular dynamics simulations. ${ }^{21,22}$ Premelting in nanosized ice/solid composites was studied by thermodynamic measurements, ${ }^{23,24} \mathrm{X}-\mathrm{ray}^{25}$ and neutron diffraction, ${ }^{26}$ quasi elastic neutron scattering, ${ }^{27} \mathrm{NMR},{ }^{28}$ and time-domain reflectometry. ${ }^{29}$ However, the liquid layer thicknesses obtained by the different experimental techniques and computer simulations differ by almost two orders of magnitude. ${ }^{5,6}$ In particular, for the class of ice composites, most studies focused on the premelting in naturally occurring samples from permafrost regions with complex composition and morphology. ${ }^{30,31}$ In general, this precludes a quantitative discussion of the intrinsic premelting mechanisms in terms of physical models. This led to the development of semi-empirical descriptions ${ }^{24}$ rather than unifying theories as discovered for premelting at free surfaces. ${ }^{32}$ Thus, experimental results on well defined model systems are essential to advance the physical understanding of interfacial premelting at buried interfaces and inside slit pore confinement.

Here, we present a high energy X-ray diffraction (XRD) study of the interfacial melting in ice/vermiculite and ice/kaolin composites. Vermiculite and kaolin are phyllosilicate clay minerals, forming planar platelets with a large aspect ratio and a large specific surface area (Fig. 2). The paper is structured as follows: In Section 2 we introduce a premelting model for water and ice inside slit pores with specified size distributions. In Section 3 we report the preparation and characterization of the nano composite materials. Well defined vermiculite samples of high purity were obtained by chemical processing of the natural mineral. This suppresses geometry and impurity driven effects. The high energy X-ray diffraction experiments and data processing are described in

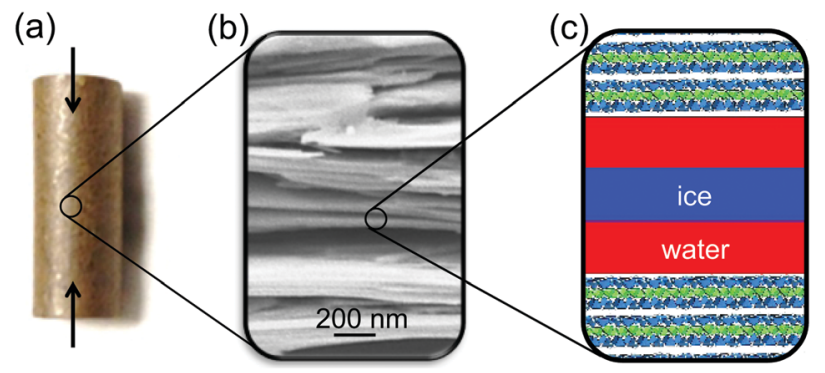

Fig. 2 (a) Photography of a compacted vermiculite $/ \mathrm{H}_{2} \mathrm{O}$ pellet. Arrows indicate the compression direction. (b) SEM image of the modified dry vermiculite. (c) Sketch of interfacial ice melting in sheet silicates (ice: blue, premelting layer: red, sketch not to scale).
Sections 3.2 and 4.1. The growth law of the effective premelting layer thickness vs. temperature, $\bar{d}(T)$, is calculated from the scattering data and clay powder properties. The quantitative analysis of the experimentally obtained growth law using the maximum entropy (MaxEnt) approach is described in Section 4.2. In Section 5 we discuss the parameters extracted from the experimental data. From comparison between the results obtained for charged vermiculite and uncharged kaolin clays we extract information on the relevant molecular interactions governing the intrinsic interfacial melting mechanism.

\section{Premelting in slit pore structures}

\subsection{Premelting at semi-infinite planar interfaces}

Near interfaces, intermolecular interactions differ from bulk. Locally, these differences can induce phase transitions such as surface melting and surface freezing. The thermodynamics of these interface induced phase transitions can be described within the framework of classical wetting theory. ${ }^{33,34}$ In the special case of interfacial premelting adjacent to a solid (s), a liquid layer (l) is wetting its crystalline ice phase (i). The growth law of the premelting layer thickness $d(T)$ is determined by the balance between the effective thickness-dependent interfacial free energy $\gamma(d)$ and the entropy of the liquid relative to the crystalline ice. ${ }^{5,33-36}$ For short-range forces the interfacial free energy $\gamma(d)$ exhibits an exponential asymptotic decay, which can be approximated by the following empirical form:

$$
\begin{gathered}
\gamma(d)=\gamma_{\mathrm{i}-\mathrm{s}}+\varphi(d) \Delta \gamma, \\
\varphi(d)=1-\mathrm{e}^{-d / \lambda} .
\end{gathered}
$$

Here, $\Delta \gamma=\gamma_{i-1}+\gamma_{1-s}-\gamma_{i-s}$ is the deviation from Antonow's rule, coupling the interfacial free energies of the bulk ice/liquid $\gamma_{i-1}$, liquid/solid $\gamma_{1-s}$, and bulk ice/solid $\gamma_{\mathrm{i}-\mathrm{s}}$ interfaces. The parameter $\lambda$ controls the decay length of the prefactor $\varphi(d)$ describing the premelting transition from a single ice-solid interface below $T_{0}$ with $\varphi(0)=0$ (Fig. 1a) to two independent ice/liquid and liquid/ solid interfaces with $\varphi(\infty)=1$ at bulk ice/liquid coexistence. This approach directly leads to a logarithmic dependence of the equilibrium layer thickness with temperature:

$$
\begin{gathered}
d(T)=\lambda \ln \left(\frac{T_{\mathrm{m}}-T_{0}}{T_{\mathrm{m}}-T}\right), \\
T_{0}=T_{\mathrm{m}}\left(1+\frac{\Delta \gamma \nu}{\Delta H \lambda}\right) .
\end{gathered}
$$

Here, $\Delta H$ is the molar latent heat of fusion and $v$ is the molar volume.

\subsection{Decay length and bulk correlations}

Based on a Landau-Ginzburg model for a non-ordering surface phase transition, Lipowsky proposed that in the generic case the decay length $\lambda$ of the surface free energy $\gamma(d)$ and the bulk correlation length $\xi$ of the liquid are identical. ${ }^{37,38}$ In eqn (2) the coupling between the (i-l) and (l-s) interfaces over the distance $d$ leading to the gradual change in the interfacial free 
energy $\gamma(d)$ is mediated via the correlations in the interfacial liquid layer. Given an effective intermolecular potential of finite range, the envelope of the two point correlation function in a bulk liquid asymptotically decays with $r^{-1} \exp (-r / \xi)$ as $r \rightarrow \infty .^{39,40}$

At surfaces and buried interfaces, as is expressed by the Yvon equation, ${ }^{40}$ these correlations drive an interfacial profile that decays with $\exp (-r / \xi) .{ }^{41}$ Hence, the decay length $\lambda$ in the growth given by eqn (3) is linked to the bulk correlation length $\xi$ of the liquid.

Information on $\xi$ is contained in the liquid structure factor. It can be determined from MD simulations, or from X-ray or neutron scattering. From high precision scattering data ${ }^{42-45}$ we extract $\lambda=\xi=2.42 \AA$ for the bulk correlation length introduced in eqn (2) (Section A, ESI $\dagger$ ). Temperature changes lead to slight variation in the $\mathrm{X}$-ray scattering patterns, ${ }^{46}$ mainly affecting the periodicity.

\subsection{Premelting in slit pores}

In contrast to semi-infinite ice/solid interfaces (Fig. 1b) premelting in clay minerals occurs in slit pores (Fig. 2). In order to model premelting in such geometries, we first consider a single pore of width $D$ in between two parallel planar solid substrates filled with water at the reduced temperature

$$
\tau=\frac{T-T_{\mathrm{m}}}{T_{\mathrm{m}}}<0
$$

Two different premelting configurations are conceivable. Firstly, the slit pore can be partially molten. In this case, layers of liquid water of thickness $d \in[0,1 / 2 D)$ cover each solid substrate (Fig. 3a). The free energy of the liquid layers $F(d)$ per substrate area $A$ is obtained from eqn (1) and (2):

$$
\frac{F(d<1 / 2 D)}{A}=\gamma(d)-\frac{d \Delta H}{v} \tau \text {. }
$$

The first term on the right-hand side of eqn (5) accounts for the free energy of the liquid/solid and the ice/liquid interfaces. The second term describes the free energy increase due to the premelting layers of bulk metastable liquid water being formed instead of bulk stable ice.

In the second case, the slit pore is completely molten i.e. $d=1 / 2 D$. Here, liquid water fills the entire pore such that no ice/liquid interfaces are present (Fig. 3b). Then the free energy per interfacial area is given by

$$
\frac{F(d=1 / 2 D)}{A}=\gamma_{1-\mathrm{s}}-\frac{D \Delta H}{2 v} \tau .
$$

(a) partially molten

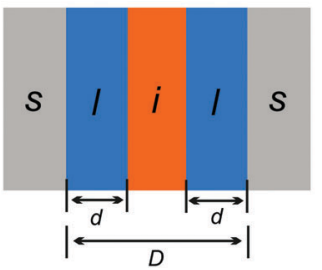

Fig. 3 Partially (a) and completely (b) molten case of liquid water (l) and ice (i) confined in a slit pore made of two solids (s) spaced by a distance $D$.
For reduced temperatures $\tau<0$, the equilibrium layer thickness

$$
\hat{d}(D, \tau)= \begin{cases}1 / 2 D, & D<D_{\mathrm{c}}(\tau) \\ d_{\mathrm{p}}(\tau), & D>D_{\mathrm{c}}(\tau)\end{cases}
$$

in pores of width $D$ is determined by the minimum of the free energy $F(d)$ given by eqn (5) and (6). The crossover between partial and complete premelting occurs at a pore width

$$
D_{\mathrm{c}}(\tau)=\left\{\begin{array}{ll}
2 \xi \frac{\tau_{0}-\Delta \tau}{\tau}, & \tau<\tau_{0} \\
2 \xi\left[\ln \left(\frac{\tau_{0}}{\tau}\right)+\frac{\tau-\Delta \tau}{\tau}\right], & \tau>\tau_{0} .
\end{array} .\right.
$$

In the case of partial premelting, an equilibrium water layer thickness

$$
d_{\mathrm{p}}(\tau)= \begin{cases}0, & \tau<\tau_{0} \\ \xi \ln \left(\frac{\tau_{0}}{\tau}\right), & \tau>\tau_{0}\end{cases}
$$

is found. Eqn (9) resembles the same logarithmic growth law obtained in eqn (3) for semi-infinite systems. It applies to all pores of width $D>D_{\mathrm{c}}(\tau)$ that are not completely molten. Unlike suggested previously, ${ }^{27}$ in a single slit pore there is no shift of the premelting curve to lower temperatures compared to the semi-infinite system described by eqn (3). However, when a critical thickness $D_{\mathrm{c}}$ is reached there is a discontinuous transition to the completely molten case.

In analogy to eqn ( $3 b)$, the reduced onset temperature

$$
\tau_{0}=\frac{T_{0}-T_{\mathrm{m}}}{T_{\mathrm{m}}}=\frac{\Delta \gamma \nu}{\Delta H \xi}
$$

is a substrate-dependent quantity as $\Delta \gamma$ contains $\gamma_{1-\mathrm{s}}$ and $\gamma_{\mathrm{i}-\mathrm{s}}$. In the present work $\tau_{0}$, and hence $\Delta \gamma$, is determined by means of a MaxEnt approach described in Section 4.2. In contrast,

$$
\Delta \tau=\frac{\gamma_{\mathrm{i}-1} v}{\Delta H \xi}>0
$$

is determined exclusively by the substance filling the pores. For the case of water, $\Delta \tau=0.396$ is obtained from $\Delta H=$ $6.01 \mathrm{~kJ} \mathrm{~mol}^{-1}, \gamma_{\mathrm{i}-1}=32 \mathrm{~mJ} \mathrm{~m}{ }^{-2}, v=18 \mathrm{~cm}^{3} \mathrm{~mol}^{-1},{ }^{11}$ and $\xi=2.42 \AA$ (Section 2.2).

To include interface coupling via long-range van der Waals forces, ${ }^{4-50}$ eqn (2) is modified. However, the procedure described here for interactions dominated by exponentially decaying effective short-range forces can be followed in an analog way.

\subsection{Pore size distribution}

For slit- or wedge-shaped pores with size distribution $P(D)$ the effective growth law $\bar{d}(T)$ of the liquid water layer thickness with temperature is expected to differ from the semi-infinite case discussed in Section 2.1. This particularly holds true for the premelting in clay minerals determined by X-ray scattering reported in Section 4.

Let $P(D)$ denote the probability density of a surface element of the substrate walls to belong to a pore of width $D$. Using eqn (7), 
the effective layer thickness $\bar{d}(T)$ at temperature $T$ can be expressed as

$$
\bar{d}(T)=\int_{0}^{\infty} \mathrm{d} D \hat{d}(D, \tau) P(D) .
$$

In Section 4.2 this relation is inverted to obtain the pore size distribution $P(D)$ from expermimetally determined effective water layer thicknesses $\bar{d}(T)$ by a MaxEnt approach.

\section{Experimental}

\subsection{Sample preparation}

Charged vermiculite nano-platelets were prepared from thermally exfoliated natural vermiculite (Sigma Aldrich) by a physiochemical process. Gross impurities were removed by iteratively dispersing the raw material in deionized water and removing dense minerals by sedimentation. A slurry of $5 \mathrm{wt} \%$ vermiculite in $\mathrm{H}_{2} \mathrm{O}_{2}$ solution (15 wt $\%$ ) was stirred at $50{ }^{\circ} \mathrm{C}$ for one hour to promote exfoliation. ${ }^{51}$ Motivated by the RCA standard cleaning procedure, ${ }^{52}$ the $\mathrm{H}_{2} \mathrm{O}_{2}$ slurry was then sonicated for $30 \mathrm{~min}$ in an alkaline environment ( $2 \mathrm{wt} \% \mathrm{NH}_{3}$ ), followed by stirring in an acidic solution (5 wt\% $\mathrm{HCl})$. The alkaline treatment further increases the surface area by zeta potential induced exfoliation of the vermiculite sheets and effectively reduces organic contaminants. The acidic treatment removes $\mathrm{Fe}^{2+}$ and $\mathrm{Mg}^{2+}$ ions from the surface. Cations at the vermiculite surface were exchanged with $\mathrm{K}^{+}$by repeated dispersion in $1 \mathrm{~mol} \mathrm{~L}^{-1} \mathrm{KCl}$ solution to limit swelling. ${ }^{53}$ Excess ions were removed by multiple subsequent dispersion in deionized water and filtering. Kaolin powder (Acros Organics) with neutral surface charge was used as received (ID31 sample) or purified by dispersion/filtering cycles (P07 sample). Properties of the different samples are summarized in Table 1.

\subsection{High energy X-ray diffraction}

The ice content in partially frozen clay composites was studied by high energy X-ray diffraction at beamlines ID31, ESRF and P07, PETRAIII. Experimental details are summarized in Table S2 $(\mathrm{ESI} \dagger)$. As an internal XRD calibration standard, $1 \mathrm{wt} \% \mathrm{CeO}_{2}$ was added to the clays. Cylindrical pellets $(6 \mathrm{~mm}$ diameter, $15 \mathrm{~mm}$ length) were prepared by uniaxially compacting the water soaked materials at approx. 100 bar (Fig. 2a). The resultant total water

Table 1 Properties of clay nano composite samples used for XRD experiments at beamline (BL) ID31, ESRF and P07, PETRA III. Mass density of the pure mineral $\rho$, specific surface area $S$, average clay platelet thickness $L$, water mass content $w_{\mathrm{m}}$, average slit pore width $\bar{D}_{\mathrm{sa}}$, and surface free ion concentration $c_{s}$

\begin{tabular}{llll}
\hline & BL & Vermiculite & Kaolin \\
\hline$\rho$ & & $2.63 \mathrm{~g} \mathrm{~cm}^{-3}$ & $2.70 \mathrm{~g} \mathrm{~cm}^{-3}$ \\
$s$ & & $36.4 \mathrm{~m}^{2} \mathrm{~g}^{-1}$ & $10.9 \mathrm{~m}^{2} \mathrm{~g}^{-1}$ \\
$L$ & & $20.9 \mathrm{~nm}^{2}$ & $68.0 \mathrm{~nm}$ \\
$w_{\mathrm{m}}$ & ID31 & $27 \mathrm{wt} \%$ & $20 \mathrm{wt} \%$ \\
& P07 & - & $12 \mathrm{wt} \%$ \\
$\bar{D}_{\mathrm{sa}}$ & ID31 & $20.3 \mathrm{~nm}$ & $45.9 \mathrm{~nm}$ \\
& P07 & - & $25.0 \mathrm{~nm}$ \\
$c_{\mathrm{s}}$ & ID31 & $0.40 \mu \mathrm{mol} \mathrm{m}$ & $0.35 \mu \mathrm{mol} \mathrm{m} \mathrm{mol}^{-2}$ \\
& P07 & - & $0.04 \mu \mathrm{mol} \mathrm{m}^{-2}$
\end{tabular}

mass content $w_{\mathrm{m}}$ of the pellets was determined in retrospect by thermogravimetric analysis (TGA/DSC 3+, Mettler Toledo) after the XRD experiments. Measurements result in $27 \mathrm{wt} \%, 20 \mathrm{wt} \%$, and $12 \mathrm{wt} \%$ for the vermiculite, ID31 and P07 kaolin samples, respectively. The pellets were placed inside a temperature controlled titanium cell with stability better than $\pm 0.005{ }^{\circ} \mathrm{C}$. To increase thermal contact and to avoid water evaporation during the measurements, the cell was filled with 2,2,4-trimethylpentane. Scattered intensities were recorded on area detectors. For each temperature, at least two series of 117 independent 2D-scattering patterns were collected by rotating and translating the sample along the cylinder axes. This procedure allowed the collection of representative and reproducible XRD averages. After rapidly cooling the sample below $-60{ }^{\circ} \mathrm{C}$ and equilibration for $40 \mathrm{~min}$, XRD measurements were made while stepwise increasing the temperature until complete melting was observed.

\section{Analysis and results}

\subsection{High energy X-ray diffraction}

The 2D-XRD scattering patterns from kaolin and vermiculite samples measured at different sample positions were normalized by their integrated $\mathrm{CeO}_{2}$ (111) Bragg intensities and averaged (Fig. S6, ESI $\dagger$ ). Pronounced textures are observed in the clay Bragg reflections. For the vermiculite (002), kaolin (001), and kaolin (002) reflections, maxima are observed in the vertical direction, i.e. parallel to the apex of the cylindrical pellet. This is explained by the parallel alignment of the platelets during the pellet preparation by uniaxial compression (Fig. 2). No singular intense Bragg spots were observed in the 2D-XRD patterns from ice. This indicates the absence of large ice single crystals after rapid freezing of the composite samples. Therefore, the integrated intensity of the Bragg reflections provides a quantitative measure of the ice content.

After azimuthal integration, high temperature scattering patterns of the molten samples were subtracted from each temperature set (Fig. 4, Section G, ESI $\dagger$ ). This procedure efficiently eliminates most of the scattering signal from the clay minerals, $\mathrm{CeO}_{2}$ reference, and sample container. Thermal expansion leads to S-shaped curves in the difference patterns in the region around the kaolin (002) and (111), vermiculite (021), and $\mathrm{CeO}_{2}$ (111) Bragg reflections.

Integrated Bragg intensities of ice Ih were determined from simultaneously fitting three Gaussian peaks for the ice (100), (002), and (101) Bragg reflections to the corrected azimuthally integrated XRD difference patterns (Tables S3-S5, ESI $\dagger$ ). The positions of the Bragg reflections agree well with literature values for the ice lattice spacings and thermal expansion. ${ }^{54}$ The peaks' FWHM are limited by the angular resolution of the experimental setup using large pellet samples of $6 \mathrm{~mm}$ diameter. Therefore, details in the line shape of the Bragg reflections are concealed.

Below the bulk melting point $T_{\mathrm{m}}$, the intensities $I(T)$ of the (100), (002), and (101) ice Ih Bragg reflections rapidly increase (Fig. 4). This corresponds to a decreasing liquid water fraction (Fig. S8, ESI $\dagger$ ) that is related to the effective premelting layer 


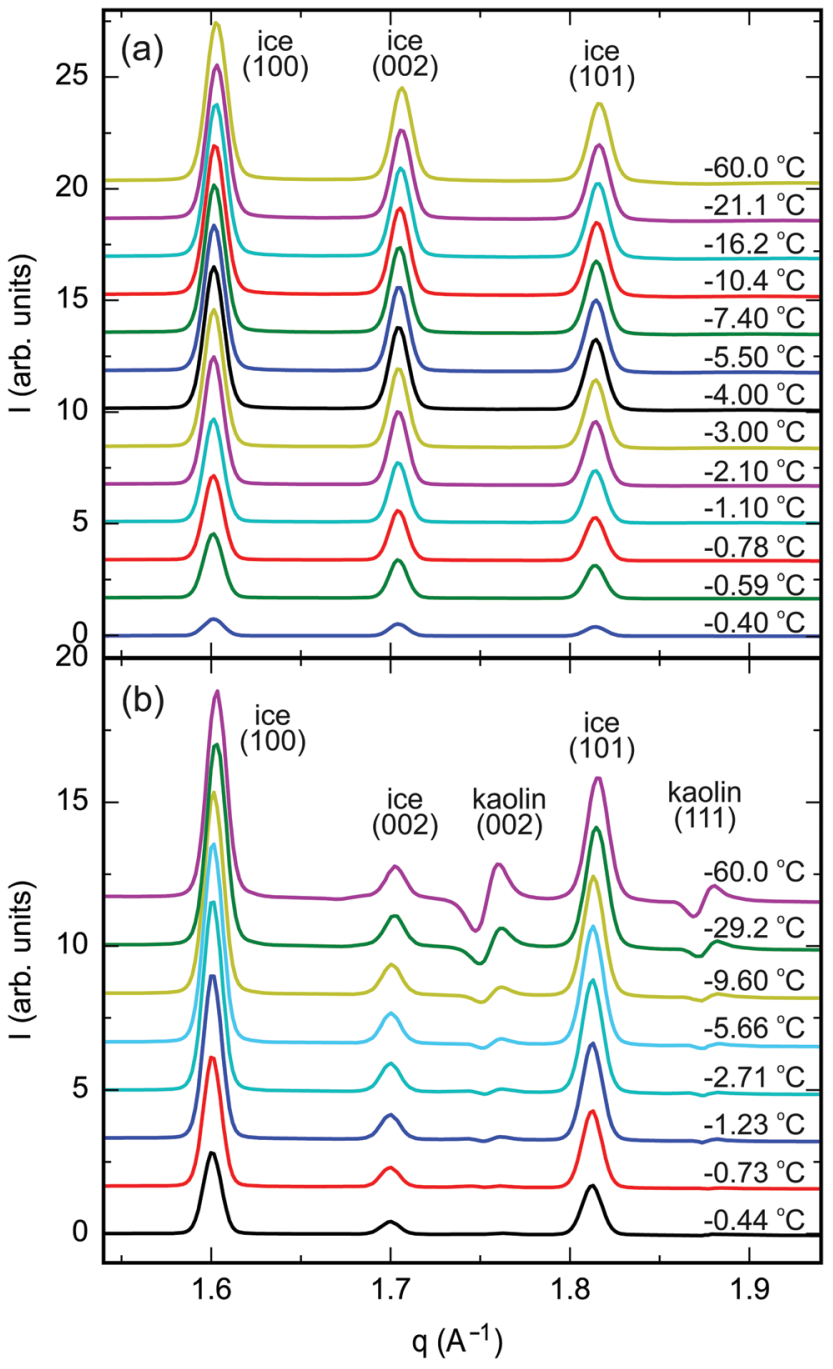

Fig. 4 Azimuthally integrated XRD difference patterns between partially frozen samples at $-60{ }^{\circ} \mathrm{C}<T-T_{m}<0{ }^{\circ} \mathrm{C}$ and the molten samples above $T_{m}$; (a) vermiculite, (b) kaolin (ID31, ESRF).

thickness $\bar{d}$ (Section G, ESI $\dagger$ ). Fig. 5 shows $\bar{d}$ vs. temperature below melting, $T_{\mathrm{m}}-T$. Upon cooling, a rapid decrease of $\bar{d}$ is observed for all samples.

\subsection{MaxEnt analysis}

In this section, we estimate the pore size distribution $P(D)$ of a sample from a set of liquid water layer thicknesses $\bar{d}_{i}=\bar{d}\left(T_{i}\right), i \in$ $\{1, \ldots, n\}$, measured at $n$ temperatures $T_{i}$ (Section 4.1).

The functional form of the pore size distribution $P(D)$ is constrained by the normalization condition

$$
\int_{0}^{\infty} \mathrm{d} D P(D)=1
$$

and the values of the experimental data (eqn (12)):

$$
\int_{0}^{\infty} \mathrm{d} D \hat{d}\left(D, \tau_{i}\right) P(D)=\bar{d}_{i}, \quad i \in\{1, \ldots, n\} .
$$

Note that $\hat{d}(D, \tau)$ in eqn (7) additionally depends on the $a$ priori unknown reduced onset temperature $\tau_{0}$.

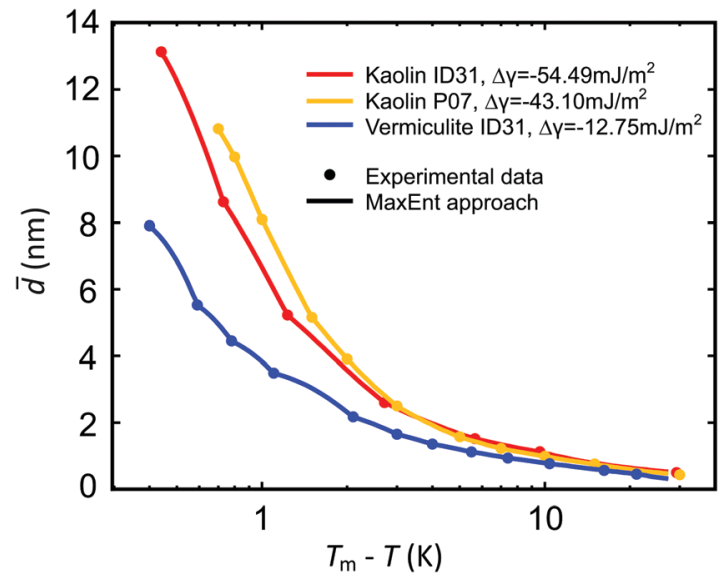

Fig. 5 Effective premelting layer thickness $\bar{d}$ vs. temperature below melting, $T_{m}-T$, for vermiculite (blue) and kaolin (ID31: red; P07: orange) composite samples extracted from the intensity of the ice (100), (002), (101) Bragg reflections (dots). Solid curves are calculated by eqn (2) with pore size distributions $P(D)$ obtained from a MaxEnt analysis (Section 4.2 and Fig. 6).

The MaxEnt method yields the least biased distribution $P(D)$ compatible with the boundary conditions eqn (13) and (14) by constrained maximization of a certain entropy functional $H[\tilde{P}]$ on the set of all distributions $\tilde{P}(D) \cdot{ }^{5,56}$ For the present case of linear constraints, the relevant functional is given by ${ }^{57}$

$$
H[\tilde{P}]=-\int_{0}^{\infty} \mathrm{d} D \tilde{P}(D) \ln \left[\frac{\tilde{P}(D)}{P_{0}(D)}\right]
$$

with $P_{0}(D)$ representing the a priori knowledge of the pore size distribution, i.e., without taking the measurement data $\bar{d}_{i}, i \in$ $\{1, \ldots, n\}$ into account. Here $P_{0}(D)$ is the uniform distribution in the range $D \in\left[0, D_{\max }\right]$

$$
P_{0}(D)= \begin{cases}D_{\max }^{-1}, & D<D_{\max } \\ 0, & D>D_{\max }\end{cases}
$$

with maximum pore size $D_{\max }$ (Table 2). As consequence, integrals in eqn (13)-(15) can be limited to the range $D \in\left[0, D_{\max }\right]$.

The entropy functional $H[\tilde{P}]$ was maximized under the constraints eqn (13) and (14) with respect to $\tilde{P}(D)$ and $\tau_{0}$ using the method of Lagrange multipliers. For all samples, the effective premelting layer thicknesses $\bar{d}(T)$ (Fig. 5, solid lines) calculated from the resultant pore size distributions $P(D)$ (Fig. 6) reproduce the experimentally observed values (Fig. 5 , symbols). The value of $\Delta \gamma$ for each individual sample is calculated from $\tau_{0}$ via eqn (10). Parameters for the reduced onset temperature $\tau_{0}$, the deviation

Table 2 Parameters for reduced onset temperature $\tau_{0}$, deviation from Antonow's rule $\Delta \gamma$, and average slit pore width $\bar{D}_{\text {psd }}$ obtained for a cut-off width $D_{\max }$ within the MaxEnt approach of Section 4.2 for the three clay nano composite samples characterized in Table 1

\begin{tabular}{lllll}
\hline Sample & $\tau_{0}$ & $\Delta \gamma\left(\mathrm{mJ} \mathrm{m}^{-2}\right)$ & $\bar{D}_{\mathrm{psd}}(\mathrm{nm})$ & $D_{\max }(\mathrm{nm})$ \\
\hline Vermiculite & -0.158 & -12.8 & 15.8 & 134 \\
Kaolin (ID31) & -0.675 & -54.5 & 26.2 & 123 \\
Kaolin (P07) & -0.534 & -43.1 & 21.6 & 87
\end{tabular}




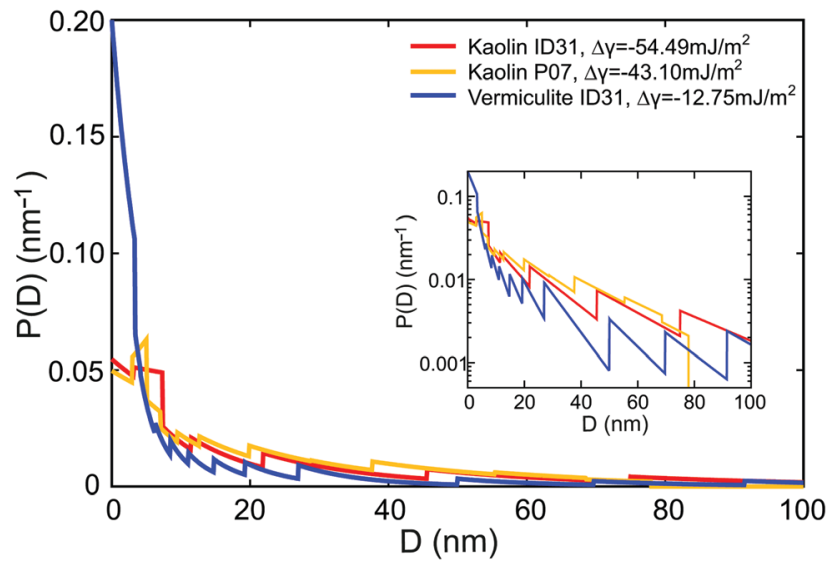

Fig. 6 Pore size distribution $P(D)$ for vermiculite (blue) and kaolin (ID31: red; P07: orange) nano composite samples extracted from the experimental premelting layer thicknesses $\bar{d}(T)$ by MaxEnt analysis. The inset shows $P(D)$ on a logarithmic scale.

from Antonow's rule $\Delta \gamma$, and the average pore size $\bar{D}_{\text {psd }}$ are summarized in Table 2.

\section{Discussion and conclusions}

In Section. 4.1, we experimentally determined the interfacial premelting of ice in two well characterized ice-nanocomposite model systems comprised of a charged and an uncharged clay. The growth of the effective premelting layer thickness $\bar{d} v s$. temperature below melting $T_{\mathrm{m}}-T$ is shown in Fig. 5 . Close to the bulk melting point, layer thicknesses approach values corresponding to half the average slit pore widths $\bar{D}_{\mathrm{psd}}$. In the low temperature region, liquid water is still detected below $-20{ }^{\circ} \mathrm{C}$. For both kaolin samples we find consistently larger $\bar{d}$ values compared to vermiculite at the same temperature. At $T_{\mathrm{m}}$ $-T=1.0 \mathrm{~K}$ the liquid fractions $f\left(T_{\mathrm{m}}-T\right)$ lie in between 0.2 and 0.4 . This corresponds to premelting layer thicknesses of $3.5 \mathrm{~nm}$ for vermiculite and $7.5 \mathrm{~nm}$ for kaolin samples. The former value is similar to the premelting layer thicknesses of $2 \mathrm{~nm}$ to $4 \mathrm{~nm}$ observed by X-ray reflectivity at the interface between single crystalline ice and amorphous $\mathrm{SiO}_{2}{ }^{16}$ and quartz. ${ }^{18}$ For graphitized carbon black and talc powders Maruyama extracted frozen fractions by quasi elastic neutron scattering. ${ }^{27}$ At $T_{\mathrm{m}}-$ $T=1.0 \mathrm{~K}$, the more hydrophobic carbon black $(f \sim 0.4)$ shows significantly larger liquid fractions compared to the sheet silicate talc $(f \sim 0.09)$. For the free ice surface even thicker premelting layers from $30 \mathrm{~nm}$ to $50 \mathrm{~nm}$ were reported by Lied ${ }^{15}$ and Döppenschmidt. ${ }^{58}$ Thus, phenomenological comparison of our results for the effective premelting layer thickness $\bar{d}$ with the literature provides a first hint for a presumably general tendency: for ice, the premelting layer thickness gets larger at more hydrophobic interfaces.

However, for a quantitative analysis and interpretation the interactions with the solid substrate governing $\Delta \gamma$ and the pore size distribution $P(D)$ have to be taken into account. A slit pore premelting model (Section 2) in conjunction with a MaxEnt method (Section 4.2) allows for a full quantitative analysis of experimentally determined growth laws $\bar{d}(T)$. Fig. 5 demonstrates that this continuum model adequately describes the experimental premelting data over the entire measured temperature range.

The pore size distribution $P(D)$, extracted by the MaxEnt analysis (Fig. 6), is an important parameter of the model. Its functional dependency exhibits a fast decay for larger $D$ values. For all clay nano composite samples, the average slit pore widths $\bar{D}_{\text {psd }}$ calculated from $P(D)$ (Table 2) are consistent with the $\bar{D}_{\mathrm{sa}}$ values determined from the gas adsorption isotherms (Table 1 ). The systematically smaller values obtained for the dry powders $\left(\bar{D}_{\mathrm{sa}}\right)$ compared to the pressed clay/water pellets $\left(\bar{D}_{\text {psd }}\right)$ are explained by their different preparation states.

For the effective coupling between the solid/liquid and liquid/ice interface we assume effective short-range forces, generating an exponentially decaying interfacial free energy $\gamma(d)$ in eqn (2). Their decay length $\lambda$ equals the bulk correlation length $\xi$ in the liquid phase. ${ }^{37}$ From bulk X-ray scattering data of liquid water, we extract $\lambda=\xi=2.42 \AA$. Indeed, our experimental premelting data is perfectly reproduced using this bulk value for all samples independent of the contained clay mineral. Thus, our results are in agreement with the standard theory of interface phase transitions. Likewise, Smit et al. found that the characteristic spectroscopic response from the ice premelting layer and supercooled bulk water is indistinguishable by sum-frequency generation spectroscopy. ${ }^{59}$ However, changes in the water structure have been observed for water confined in nano-pores ${ }^{60}$ Scattering experiments indicate that confined water exhibits a higher order compared to bulk. ${ }^{61-64}$ Likewise, the increase in premelting liquid layer density adjacent to hydrophilic $\mathrm{SiO}_{2}$ by $20 \%$ compared to bulk water ${ }^{16}$ and surface spectroscopy experiments ${ }^{20,65}$ indicate different properties. Such huge density changes would require structural changes that are much larger compared to the temperature variations observed for supercooled water. ${ }^{46}$ The interpolation of the interfacial free energy $\gamma(d)$ between $d=0$ and $d \rightarrow \infty$ given in eqn (1) and (2) obeys the thermodynamically correct exponential asymptotic decay on the scale of the bulk correlation length $\lambda=\xi$. However, from the results discussed above it is expected that the structural properties of confined water lead to deviations from this simple form. Hence, deviations from eqn (9) may occur for small water layer thicknesses $d_{\mathrm{p}}$ at low temperatures. The present work highlights the importance of the pore size distribution $P(D)$ for the premelting behavior in nano composite materials such as sheet silicates. In consequence, the specific small-thickness features of the interfacial free energy $\gamma(d)$ are expected to become relevant only for samples with a large fraction of very small pores.

The third parameter affecting premelting in slit pore confinement is the deviation from Antonow's rule $\Delta \gamma$. This quantity, related to the spreading coefficient $S=-\Delta \gamma$ of liquid water on the solid compared to ice, depends on the balance of the interactions between the three components. Both clays studied in this work form planar hydrophilic sheets (Fig. S4 and S5, ESI $\dagger$ ). While vermiculite surfaces are charged by $1.2-1.8$ unit charges per unit cell, polar kaolin surfaces are neutral. ${ }^{66}$ Therefore, different $\Delta \gamma$ values are expected to result in different premelting curves for vermiculite and kaolin based ice/clay composites. 
Indeed, about four times larger negative $\Delta \gamma$ values were extracted for kaolin compared to vermiculite by MaxEnt analysis (Table 2).

For vermiculite, we find $\Delta \gamma=-12.8 \mathrm{~mJ} \mathrm{~m}^{-2}$. For comparison, for single crystal ice $/ \mathrm{SiO}_{2}$ interfaces $\Delta \gamma=-5.03 \mathrm{~mJ} \mathrm{~m}{ }^{-2}$ is obtained from $T_{\mathrm{m}}-T_{0}=17 \mathrm{~K}$ reported by Engemann et al. ${ }^{16}$ A common property of vermiculite and $\mathrm{SiO}_{2}$ surfaces is their negative charge.

For the two kaolin samples, $\Delta \gamma$ values agree well, despite their different preparation protocols leading to different ion and water concentrations. This confirms the robustness of the MaxEnt analysis method. It also indicates that the differences in the observed growth law for kaolin and vermiculite are primarily related to the surface properties and the pore size distribution of the clay minerals rather than impurities. Bare kaolin surfaces have a surface tension of $171 \mathrm{mN} \mathrm{m}^{-1}$ with a ratio of $40 \%$ dispersive and $60 \%$ nondispersive interactions. ${ }^{67}$ The positive spreading coefficient $S=76 \mathrm{mN} \mathrm{m}^{-1}$ of liquid water $v s$. its vapor, calculated by the Fowkes method, ${ }^{68}$ reflects the strong hydrophilic nature of the bare kaolin surface. With about $50 \mathrm{~mJ} \mathrm{~m}^{-2}$ the spreading coefficient of liquid water $v s$. ice found in this work is on the same order of magnitude.

For the free ice surface, an even larger negative $\Delta \gamma$ value of $-384 \mathrm{~mJ} \mathrm{~m} \mathrm{~m}^{-2}$ is reported in the literature. ${ }^{58}$ Consistently, a large dangling hydrogen bond density was detected at the free ice surface by SFG spectroscopy. ${ }^{20,69}$ In contrast to ice, in liquid water the hydrogen bonding network adjacent to interfaces can rearrange. Therefore, the formation of a liquid premelting layer at ice surfaces is energetically favorable. This is reflected in a large negative $\Delta \gamma$ value.

Generally, larger negative $\Delta \gamma$ values indicate that the ice structure at an interface is less compatible with the opposing medium. Therefore, these media tend to be less efficient ice nucleators and vice versa. Murray et al. studied heterogeneous ice nucleation by kaolin particles in suspensions. ${ }^{70}$ Compared to other minerals, kaolin is a rather inefficient ice nucleator. ${ }^{71}$ Accordingly, we observe large premelting layer thicknesses caused by $\Delta \gamma$ values around $-50 \mathrm{~mJ} \mathrm{~m}^{-2}$. On the other hand, at quartz with a smaller absolute $\Delta \gamma=-5.03 \mathrm{~mJ} \mathrm{~m}^{-2}$, ice nucleates with a significantly higher activity. ${ }^{71}$ Likewise, ice/ vermiculite interfaces with $\Delta \gamma=-12.8 \mathrm{~mJ} \mathrm{~m}^{-2}$, exhibit thinner premelting layer thicknesses.

Premelting layer formation at ice/solid interfaces has important implications in atmospheric physics ${ }^{72}$ and geophysics. ${ }^{2}$ Our experiments on well defined ice/clay nano composite samples bridge the gap between studies on single crystalline ice/solid model interfaces ${ }^{16}$ and naturally occurring soils. ${ }^{30,31}$ Compared to naturally occurring soils, the ion concentration in the sample was reduced to investigate the influence of the surface properties of the adjacent solid. For ice/ clay nano composites, in addition to the deviation from Antonow's rule the pore size distribution is a decisive parameter determining the liquid water fraction vs. temperature. The experimental premelting data is quantitatively reproduced over the entire temperature range by a theoretical continuum model including effective short-range interactions. Thorough material characterization allows for the comparison of parameters extracted by a MaxEnt analysis with independent experimental observations.
Future theoretical and experimental work focusing on the dynamics of the water molecules in the premelting layer of ice/ clay nano composites might provide complementary information on the nature of the interfacial premelting layer.

\section{Conflicts of interest}

There are no conflicts to declare.

\section{Acknowledgements}

We thank Thomas Buslaps (ESRF), Roberto Verbeni (ESRF), and Uta Rütt (PETRA III) for their assistance during our synchrotron experiments at beamlines ID15 and ID31, ESRF-The European Synchrotron, Grenoble and at P07, PETRA III, DESY, Hamburg, a member of the Helmholtz Association (HGF), Maren Müller (MPI-P) for SEM, Helma Burg (MPI-P) and Rüdiger Berger (MPI-P) for AFM measurements, Alexei Kiselev (KIT Karlsruhe) and Hans-Jürgen Butt (MPI-P) for helpful discussions. J. M. and M. M. acknowledge the MAINZ Graduate School of Excellence, funded through the Excellence Initiative (DFG/GSC 266). H. L. was supported by the China Scholarship Council. Open Access funding provided by the Max Planck Society.

\section{References}

1 A.-M. Kietzig, S. G. Hatzikiriakos and P. Englezos, J. Appl. Phys., 2010, 107, 081101.

2 P. J. Williams, in The Freezing of Soils: Ice in a Porous Medium and Its Environmental Significance, ed. J. S. Wettlaufer, J. G. Dash and N. Untersteiner, Springer, Heidelberg, 1999, pp. 219-239.

3 M. Faraday, Philos. Mag., 1859, 17, 162-169.

4 R. Rosenberg, Phys. Today, 2005, 58, 50-55.

5 J. G. Dash, A. W. Rempel and J. S. Wettlaufer, Rev. Mod. Phys., 2006, 78, 695-741.

6 Y. Li and G. A. Somorjai, J. Phys. Chem. C, 2007, 111, 9631-9637.

7 H. Hansen-Goos and J. S. Wettlaufer, Phys. Rev. E: Stat., Nonlinear, Soft Matter Phys., 2010, 81, 031604.

8 D. Pan, L.-M. Liu, B. Slater, A. Michaelides and E. Wang, ACS Nano, 2011, 5, 4562-4569.

9 Y. Suzuki, H. Duran, M. Steinhart, M. Kappl, H.-J. Butt and G. Floudas, Nano Lett., 2015, 15, 1987-1992.

10 J. W. Cahn, J. G. Dash and H. Fu, J. Cryst. Growth, 1992, 123, 101-108.

11 K. Morishige and K. Kawano, J. Chem. Phys., 1999, 110, 4867-4872.

12 G. H. Findenegg, S. Jähnert, D. Akcakayiran and A. Schreiber, ChemPhysChem, 2008, 9, 2651-2659.

13 J. S. Wettlaufer, Phys. Rev. Lett., 1999, 82, 2516-2519.

14 Y. Yang, M. Asta and B. B. Laird, Phys. Rev. Lett., 2013, 110, 096102.

15 A. Lied, H. Dosch and J. H. Bilgram, Phys. Rev. Lett., 1994, 72, 3554-3557. 
16 S. Engemann, H. Reichert, H. Dosch, J. Bilgram, V. Honkimäki and A. Snigirev, Phys. Rev. Lett., 2004, 92, 205701.

17 M. Mezger, S. Schöder, H. Reichert, H. Schröder, J. Okasinski, V. Honkimäki, J. Ralston, J. Bilgram, R. Roth and H. Dosch, J. Chem. Phys., 2008, 128, 244705.

18 S. Schöder, H. Reichert, H. Schröder, M. Mezger, J. S. Okasinski, V. Honkimäki, J. Bilgram and H. Dosch, Phys. Rev. Lett., 2009, 103, 095502.

19 H. Bluhm, D. F. Ogletree, S. F. Charles, H. Zahid and S. Miquel, J. Phys.: Condens. Matter, 2002, 14, L227-L233.

20 X. Wei, P. B. Miranda and Y. R. Shen, Phys. Rev. Lett., 2001, 86, 1554-1557.

21 M. M. Conde, C. Vega and A. Patrykiejew, J. Chem. Phys., 2008, 129, 014702.

22 D. T. Limmer and D. Chandler, J. Chem. Phys., 2014, 141, 18 C505.

23 T. Kozlowski, Cold Reg. Sci. Technol., 2004, 38, 93-101.

24 T. Kozlowski, Cold Reg. Sci. Technol., 2007, 49, 226-236.

25 D. M. Anderson and P. Hoekstra, Science, 1965, 149, 318-319.

26 J. M. Gay, J. Suzanne, J. G. Dash and H. Fu, J. Cryst. Growth, 1992, 125, 33-41.

27 M. Maruyama, M. Bienfait, J. G. Dash and G. Coddens, J. Cryst. Growth, 1992, 118, 33-40.

28 T. Ishizaki, M. Maruyama, Y. Furukawa and J. G. Dash, J. Cryst. Growth, 1996, 163, 455-460.

29 K. Watanabe and T. Wake, Cold Reg. Sci. Technol., 2009, 59, 34-41.

30 A. R. Tice, D. M. Anderson and K. F. Sterrett, Eng. Geol., 1981, 18, 135-146.

31 A. R. Tice, P. B. Black and R. L. Berg, Cold Reg. Sci. Technol., 1989, 17, 103-111.

32 B. F. Henson and J. M. Robinson, Phys. Rev. Lett., 2004, 92, 246107.

33 S. Dietrich, in Wetting Phenomena in Phase Transitions and Critical Phenomena, ed. C. Domb and J. Lebowitz, Academic Press, London, 1988, pp. 1-218.

34 M. Schick, in Introduction toWetting Phenomena, ed. J. Charvolin, J. F. Joanny and J. Zinn-JustinNorth-Holland, Amsterdam, 1990, pp. $415-497$.

35 R. Lipowsky, Phys. Rev. Lett., 1982, 49, 1575-1578.

36 J. G. Dash, F. Haiying and J. S. Wettlaufer, Rep. Prog. Phys., 1995, 58, 115-167.

37 R. Lipowsky, U. Breuer, K. C. Prince and H. P. Bonzel, Phys. Rev. Lett., 1989, 62, 913-916.

38 J. F. van der Veen, Surf. Sci., 1999, 433, 1-11.

39 G. A. Martynov, Fundamental Theory of Liquids, Hilger, Bristol, 1992.

40 J.-P. Hansen and I. R. McDonald, Theory of Simple Liquids: With Applications to Soft Matter, Academic Press, Oxford, 2013.

41 M. Mezger, R. Roth, H. Schröder, P. Reichert, D. Pontoni and H. Reichert, J. Chem. Phys., 2015, 142, 164707.

42 G. Hura, J. M. Sorenson, R. M. Glaeser and T. Head-Gordon, J. Chem. Phys., 2000, 113, 9140-9148.
43 A. Soper, Chem. Phys., 2000, 258, 121-137.

44 T. Head-Gordon and G. Hura, Chem. Rev., 2002, 102, 2651-2670.

45 A. Soper, J. Phys.: Condens. Matter, 2007, 19, 335206.

46 L. B. Skinner, C. Benmore, J. C. Neuefeind and J. B. Parise, J. Chem. Phys., 2014, 141, 214507.

47 D.-M. Zhu and J. Dash, Phys. Rev. Lett., 1988, 60, 432-435.

48 B. Pluis, T. Taylor, D. Frenkel and J. Van der Veen, Phys. Rev.

B: Condens. Matter Mater. Phys., 1989, 40, 1353-1356.

49 H. Löwen, T. Beier and H. Wagner, EPL, 1989, 9, 791-796.

50 L. A. Wilen, J. S. Wettlaufer, M. Elbaum and M. Schick, Phys.

Rev. B: Condens. Matter Mater. Phys., 1995, 52, 12426-12433.

51 C. Marcos and I. Rodríguez, Appl. Clay Sci., 2014, 87, 219-227.

52 W. Kern, J. Electrochem. Soc., 1990, 137, 1887-1892.

53 O. Gjems, Clay Miner. Bull., 1963, 5, 183-193.

54 K. Röttger, A. Endriss, J. Ihringer, S. Doyle and W. Kuhs, Acta Crystallogr., Sect. B: Struct. Sci., 2012, 68, 91.

55 E. T. Jaynes, Probability Theory: The Logic of Science, Cambridge University Press, 2003.

56 D. Sivia and J. Skilling, Data Analysis: A Bayesian Tutorial, Oxford University Press, Oxford, 2006.

57 J. Shore and R. Johnson, IEEE Trans. Inf. Theory, 1980, 26, 26-37.

58 A. Döppenschmidt and H.-J. Butt, Langmuir, 2000, 16, 6709-6714.

59 W. J. Smit and H. J. Bakker, Angew. Chem., 2017, 129, 15746-15750.

60 M. Erko, G. Findenegg, N. Cade, A. Michette and O. Paris, Phys. Rev. B: Condens. Matter Mater. Phys., 2011, 84, 104205.

61 J. Dore, Chem. Phys., 2000, 258, 327-347.

62 M. Ricci, F. Bruni, P. Gallo, M. Rovere and A. Soper, J. Phys.: Condens. Matter, 2000, 12, A345-A350.

63 M. Bellissent-Funel, J. Phys.: Condens. Matter, 2001, 13, 9165.

64 T. Yamaguchi, H. Hashi and S. Kittaka, J. Mol. Struct., 2006, 129, 57-62.

65 M. A. Sánchez, T. Kling, T. Ishiyama, M.-J. van Zadel, P. J. Bisson, M. Mezger, M. N. Jochum, J. D. Cyran, W. J. Smit and H. J. Bakker, Proc. Natl. Acad. Sci. U. S. A., 2017, 114, 227-232.

66 I. Bihannic, A. Delville, B. Demé, M. Plazanet, F. Villiéras and L. J. Michot, in Clay Swelling: New Insights from NeutronBased Techniques, ed. L. Liang, R. Rinaldi and H. Schober, Springer, New York, 2009, pp. 521-546.

67 E. Chibowski and P. Staszczuk, Clays Clay Miner., 1988, 36, 455-461.

68 F. M. Fowkes, Ind. Eng. Chem., 1964, 56, 40-52.

69 X. Wei, P. B. Miranda, C. Zhang and Y. Shen, Phys. Rev. B: Condens. Matter Mater. Phys., 2002, 66, 085401.

70 B. Murray, S. Broadley, T. Wilson, J. Atkinson and R. Wills, Atmos. Chem. Phys., 2011, 11, 4191-4207.

71 J. D. Atkinson, B. J. Murray, M. T. Woodhouse, T. F. Whale, K. J. Baustian, K. S. Carslaw, S. Dobbie, D. OSullivan and T. L. Malkin, Nature, 2013, 498, 355-358.

72 T. Peter, Annu. Rev. Phys. Chem., 1997, 48, 785-822. 